3D simulations of gas puff effects on edge plasma and ICRF coupling in JET

This content has been downloaded from IOPscience. Please scroll down to see the full text.

2017 Nucl. Fusion 57056042

(http://iopscience.iop.org/0029-5515/57/5/056042)

View the table of contents for this issue, or go to the journal homepage for more

Download details:

IP Address: 157.193.64.83

This content was downloaded on 08/05/2017 at 16:16

Please note that terms and conditions apply.

You may also be interested in:

3D simulations of gas puff effects on edge density and ICRF coupling in ASDEX Upgrade

W. Zhang, V. Bobkov, T. Lunt et al.

Maximization of ICRF power by SOL density tailoring with local gas injection

P. Jacquet, M. Goniche, V. Bobkov et al.

Modelling of the ICRF induced ExB convection in the scrape-off-layer of ASDEX Upgrade

W Zhang, Y Feng, J-M Noterdaeme et al.

Optimization of ICRH for core impurity control in JET-ILW

E. Lerche, M. Goniche, P. Jacquet et al.

Influence of gas puff location on the coupling of lower hybrid waves in JET ELMy H-mode plasmas

A Ekedahl, V Petrzilka, Y Baranov et al.

Chapter 4: Power and particle control

A. Loarte, B. Lipschultz, A.S. Kukushkin et al.

On the challenge of plasma heating with the JET metallic wall

M.-L. Mayoral, V. Bobkov, A. Czarnecka et al.

Impurity production from the ion cyclotron resonance heating antennas in JET

A Czarnecka, F Durodié, A C A Figueiredo et al.

$\underline{\mathrm{LH}}$ wave coupling in plasmas

V Pericoli Ridolfini, A Ekedahl, S K Erents et al. 


\title{
3D simulations of gas puff effects on edge plasma and ICRF coupling in JET
}

\author{
W. Zhang ${ }^{1,2,3}$, P. Jacquet ${ }^{4}$, E. Lerche ${ }^{4,5}$, R. Bilato ${ }^{2}$, V. Bobkov ${ }^{2}$, D. Coster ${ }^{2}$, \\ Y. Feng ${ }^{2}$, C. Guillemaut ${ }^{6}$, M. Goniche ${ }^{7}$, D. Harting ${ }^{4}$, T. Lunt ${ }^{2}$, \\ J.-M. Noterdaeme ${ }^{1,2}$, G. Szepesi ${ }^{4}$, D. Van Eester ${ }^{5}$ and the JET Contributors ${ }^{8, a}$ \\ 1 Applied Physics Department, University of Ghent, Ghent, Belgium \\ 2 Max-Planck-Institut für Plasmaphysik, Garching/Greifswald, Germany \\ ${ }^{3}$ Institute of Plasma Physics, Chinese Academy of Sciences, Hefei, People's Republic of China \\ ${ }^{4}$ CCFE, Culham Science Centre, Abingdon, OX14 3DB, United Kingdom \\ ${ }^{5}$ LPP-ERM/KMS, EUROfusion Consortium Member, Trilateral Euregio Cluster, Brussels, Belgium \\ ${ }^{6}$ Instituto de Plasmas e Fusão Nuclear, Instituto Superior Técnico, Universidade de Lisboa, Lisboa, Portugal \\ 7 CEA, IRFM, F-13108 Saint-Paul-Lez-Durance, France \\ ${ }^{8}$ EUROfusion Consortium, JET, Culham Science Centre, Abingdon, OX14 3DB, United Kingdom \\ E-mail: wei.zhang@ipp.mpg.de
}

Received 13 September 2016, revised 24 February 2017

Accepted for publication 21 March 2017

Published 7 April 2017

\begin{abstract}
Recent JET (ITER-Like Wall) experiments have shown that the fueling gas puffed from different locations of the vessel can result in different scrape-off layer (SOL) density profiles and therefore different radio frequency (RF) coupling. To reproduce the experimental observations, to understand the associated physics and to optimize the gas puff methods, we have carried out three-dimensional (3D) simulations with the EMC3-EIRENE code in JET-ILW including a realistic description of the vessel geometry and the gas injection modules (GIMs) configuration. Various gas puffing methods have been investigated, in which the location of gas fueling is the only variable parameter. The simulation results are in quantitative agreement with the experimental measurements. They confirm that compared to divertor gas fueling, mid-plane gas puffing increases the SOL density most significantly but locally, while top gas puffing increases it uniformly in toroidal direction but to a lower degree. Moreover, the present analysis corroborates the experimental findings that combined gas puff scenarios - based on distributed main chamber gas puffing — can be effective in increasing the RF coupling for multiple antennas simultaneously. The results indicate that the spreading of the gas, the local ionization and the transport of the ionized gas along the magnetic field lines connecting the local gas cloud in front of the GIMs to the antennas are responsible for the enhanced SOL density and thus the larger RF coupling.
\end{abstract}

Keywords: gas puffing, ICRF coupling, 3D simulation, edge plasma, JET

(Some figures may appear in colour only in the online journal)

\section{Introduction}

Plasma heating with electromagnetic waves in the ion cyclotron range of frequencies (ICRF) is one of the most promising auxiliary

a See the author list of 'Overview of the JET results in support to ITER' by $\mathrm{X}$. Litaudon et al to be published in Nuclear Fusion Special issue: overview and summary reports from the 26th Fusion Energy Conference (Kyoto, Japan, 17-22 October 2016). plasma heating methods in magnetic controlled fusion devices. It has been successfully implemented in present-day tokamaks and stellarators and will be applied in future fusion machines such as ITER and DEMO. As the biggest tokamak in operation at present, joint European torus (JET) is equipped with a robust ICRF heating system-four 4-strap A2 ICRF antennas (powered per pair) and one ITER-like-antenna (ILA) — capable of providing a total radio frequency power of 8.0 MW in ELMy H-Mode plasmas [1]. For 


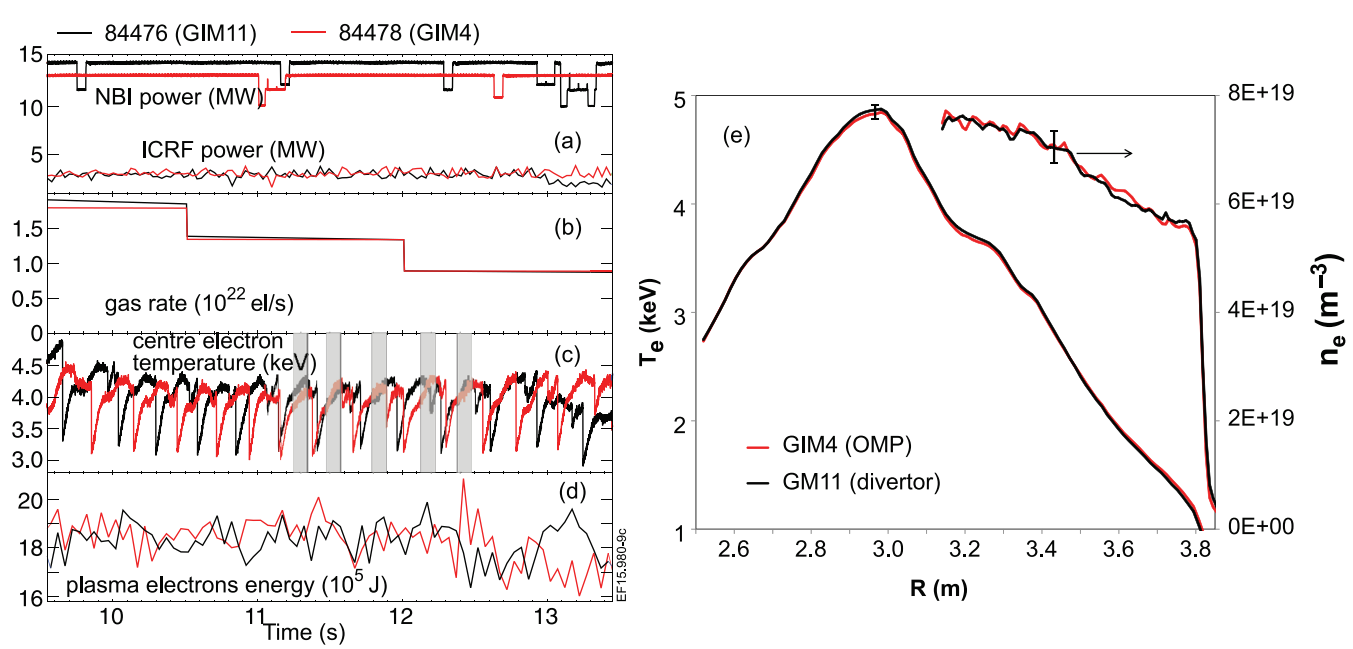

Figure 1. JET time traces and electron kinetic profiles when using divertor or OMP gas injection in JET. The time traces are $(a)$ the auxiliary heating power, $(b)$ the $D_{2}$ gas injection rate, $(c)$ the electron temperature at the plasma centre, and $(d)$ the total electron energy. Details of the Te (left $y$-axis) and ne (right $y$-axis) profiles are shown in $(e)$. The profiles are averaged over the time intervals indicated in grey on the time trace graphs. Error bars correspond to the scattering in the data. Reproduced courtesy of IAEA. Figure from [5]. Copyright 2016 IAEA.

ITER, the ICRF heating system being developed is designed to provide a total heating power of $20 \mathrm{MW}$ [2].

ICRF heating relies on the fast wave to transfer the radio frequency $(\mathrm{RF})$ power launched from the antennas into the plasma. However, the ICRF power coupling depends critically on the width of the evanescent layer $R_{\text {evan }}$ in front of the launchers, namely on the distance between the fast wave cut-off density layer and the antenna straps [3]. The amount of ICRF power coupled to the plasma can be expressed as $P_{\text {coupled }} \propto V_{\max }^{2} R_{\mathrm{c}} / 2 Z_{\mathrm{c}}^{2}$, where $V_{\max }$ is the anti-node voltage in the transmission line which is usually limited by arcing in it, $Z_{\mathrm{c}}$ is the characteristic impedance of the transmission line. Given $V_{\max }$ and $Z_{\mathrm{c}}$ values, $P_{\text {coupled }}$ is only proportional to the coupling resistance $R_{\mathrm{c}}$. However, the coupling resistance decreases exponentially with $R_{\text {evan }}$, i.e. $R_{\mathrm{c}} \propto R_{0} \mathrm{e}^{-\alpha \cdot R_{\text {evan }}}$, where $\alpha$ is a tunneling factor that mainly depends on the antenna properties (e.g. phasing) [4]. Thus, to ensure the best performances of the ICRF heating system, it is crucial to minimize this evanescent distance by increasing the plasma density in front of the antennas to a level above the fast wave cut-off density. In JET H-mode plasmas the fast wave cut-off density is typically $2.0 \times 10^{18} \mathrm{~m}^{-3}$ for dipole phasing operation [5].

The local scrape-off Layer (SOL) density can be tailored by changing the gas puff locations and the gas puff rate. Previous experiments and simulations indicate that the SOL density can be increased with main chamber (top or mid-plane) gas puffing instead of divertor gas puffing [5-10]. A pertinent question related to the use of local gas to improve ICRF wave coupling is the impact of this technique on the plasma parameters. This was discussed in some detail in [5], and repeated here for completeness. The measured plasma core kinetic profiles (Te and ne) were not modified whether divertor, top or OMP gas was used. This is illustrated in figure 1 , where the time trace of the electron temperature at the plasma centre, and electron plasma energy are plotted for pulses with divertor and OMP gas injection respectively. The electron temperature and electron density profiles for these two pulses are plotted in figure 1(e). Profiles are taken before the sawtooth crashes; data are averaged over five time windows of $0.1 \mathrm{~s}$ each as shown in figure $1(c)$. In these plasma conditions, fast wave heating results predominantly to an electron heating via slowing down of the fast $\mathrm{H}$ ions, with a typical centre electron temperature response of $\sim 0.5 \mathrm{keV} \mathrm{MW}^{-1}$. The electron kinetic profiles overlay perfectly, suggesting that within measurement errors bars, the fast wave propagation and absorption characteristics as well as the transport properties are the same, independent of the gas injection location.

An important issue when considering gas injection from different locations is the consequence of using main chamber instead of divertor gas on plasma energy confinement: In JET no degradation of pedestal pressure (figure $1(d)$ ) and confinement was observed when using the mid-plane or top inlets instead of the divertor inlets at the same gas fuelling level [5]. Another illustration is show in figure 2 where, for the series of pulse studied in this paper, the plasma energy if plotted as a function of the injection rate for different gas valve locations, showing that plasma energy was not affected by gas location. Note that in this experiment the main purpose was to measure the antenna coupling resistance reliably: the ICRH power was constant from pulse to pulse (launched-power was a set-point in a ICRF system control loop) and the power level was modest ( $\sim 3 \mathrm{MW})$; hence increase of antenna coupling resistance when using local gas puffing did not lead to higher ICRF power into the plasma, but rather it reduced the voltage at the antennas.

The quantity of gas required for antenna coupling improvement is also a key factor, particularly since increasing gas dosing can have a negative impact on plasma energy confinement in existing tokamaks [11]. In this context it is important to emphasize that the antenna coupling improvements reported here can be obtained when using moderate gas rates: in practice, the gas puff rate used in scenario development aiming at best $D-T$ target plasmas is between $0.5 \times 10^{22}$ electrons $\mathrm{s}^{-1}$ (hybrid) and $2 \times 10^{22}$ electrons $\mathrm{s}^{-1}$ (baseline) [12], while the improvement in the antenna loading in dedicated ICRF coupling experiments was $\sim 50 \%$ when fueling the plasma 


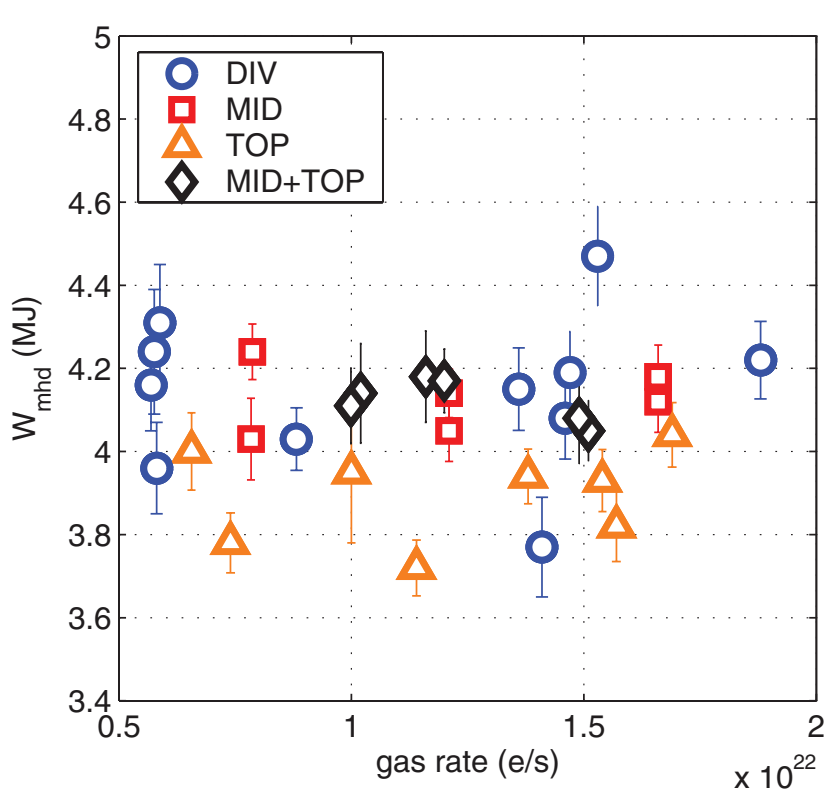

Figure 2. Plasma energy evaluated from EFIT for the series of pulses with divertor, mid-plane or top gas analysed in this paper. Note most of the $\mathrm{W}_{\text {mhd }}$ values for top puffing (6 out of the 8) were generated at slightly lower plasma current of $I_{\mathrm{p}}=2.4 \mathrm{MA}$ instead of $2.5 \mathrm{MA}$.

only with nearby mid-plane inlets instead of divertor inlets at an injection rate of $1.0 \times 10^{22}$ electrons $\mathrm{s}^{-1}$ [5]. Hence the local gas injection technique to optimize ICRF heating performance is now routinely used in JET in the development of plasma scenarios.

The success of experiments and simulations kindled the interest in enhancing ICRF coupling via local gas puffing in many tokamaks, such as ASDEX Upgrade (AUG) [7-9], DIII-D [13, 14], EAST, JET [5, 10], KSTAR, WEST [15], ITER $[16,17]$ and DEMO. For ITER in particular, the width of the evanescent layer $R_{\text {evan }}$ is larger than the one in present and past machines [10]. Moreover, the SOL density has large uncertainties in different plasma scenarios, making it difficult to make accurate predictions of the total RF coupled power. It is thus extremely important to investigate how to tailor the SOL density in front of the ICRF antennas through the local gas puffing methods and to have a suite of numerical tools validated against present experiments.

This paper mainly concentrates on the numerical studies of deuterium gas injection from different locations in JET-ILW while various experimental data have been used for comparisons. Several aspects were considered to be important in describing accurately the gas puff experiments using numerical simulations. First of all, the simulations have to be $3 \mathrm{D}$ since local gas puffing induces toroidal and poloidal inhomogeneous densities in SOL. Second, the neutrals transport and ionization by electron impact and plasma transport have to be computed accurately in realistic geometries. Third, the main chamber plasma facing components have to be taken into account because they play an important role in generating the recycling fluxes and acting as boundary conditions. Fourth, the gas valves and gas pumps need to be described realistically. EMC3-EIRENE [18] is a 3D edge plasma fluid and neutral transport code which fulfills all the requirements mentioned above. The paper is dedicated to the applications of the EMC3-EIRENE simulations in JET. To calculate the coupling resistances in different gas puffing scenarios, simulations via combining the EMC3-EIRENE simulations and the antenna code simulations are necessary. This only requires the import of the $3 \mathrm{D}$ density and temperature profiles from the EMC3-EIRENE simulations into the antenna codes.

In addition, this work is part of a continuous effort to validate the EMC3-EIRENE code using available data from present tokamaks in order to carry out future simulations for ITER with confidence and to define best gas injection locations to optimize ICRF antenna coupling. This work completes the already published simulations for AUG [8]. AUG is a middle sized tokamak with major radius/minor radius $=1.65 \mathrm{~m} / 0.5$ $0.8 \mathrm{~m}$, plasma volume $=13 \mathrm{~m}^{3}$ and $B_{\mathrm{T}} / I_{\mathrm{p}}=2.5 \mathrm{~T} / 0.8$ MA. JET is a larger tokamak with major radius/minor radius $=2.96 \mathrm{~m} / 1.25-2.10 \mathrm{~m}$, plasma volume $=100 \mathrm{~m}^{3}$ and $B_{\mathrm{T}} / I_{\mathrm{p}}=2.7 \mathrm{~T} / 2.5 \mathrm{MA}$. A full-tungsten wall is used in AUG while an ITER-like wall (tungsten divertor and Beryllium main chamber wall) is used In JET. Both in these two machines, the divertor, the mid-plane and the top gas puffing methods are investigated. The gas valve settings are different in the two machines. For instance, in AUG the top gas is usually puffed from a gas valve located in the inner top of the vessel and the resulting gas is rather localized. However, in JET the top gas is puffed right from the top of the vessel and the generated gas is poloidal widely spread. Compared to the previous work on AUG [8], the following differences/advancements are made in the paper: (1) inclusion of a divertor gas pump in the simulation model; (2) more elaborated settings of the top and midplane gas valves; (3) comparisons of the experimental and simulated neutral pressures; (4) studying the influences of the transport parameters on the ICRF coupling; (5) investigations of the combined gas puffing.

Note that density modifications induced by the ICRF induced $\mathrm{E} \times \mathrm{B}$ drifts $[19,20]$ and ponderomotive expulsion [21] are not included in the EMC3-EIRENE code. Moreover, no ionization induced by ICRF wave $[22,23]$ is considered in our simulations. In fact, evidences of RF ionization are only found when the antenna power is lower than $20 \mathrm{~kW}$ in AUG [24].

It is worth mentioning that local gas puffing can, in addition to improving the antenna coupling, also decrease the impurity influxes from antennas [7]. Changing the local density can also potentially modify the heat loads on the antenna structure, as attest scaling laws from JET experiments both with C-wall [25] and ITER-like wall [26], where it was found experimentally that heat fluxes increase with the local density in front of the antennas and the RF voltage in the transmission lines feeding the antennas. It is however observed in practice that local gas injection is also beneficial on JET to reduce the heat loads at the limiters close to the antennas. The reason might be of two folds: (a) The density increase in front of the antenna and coupling improvement reduces the antenna parallel electric field that drives RF sheaths rectification and RF specific heat loads. (b) Gas injection could also locally reduce 

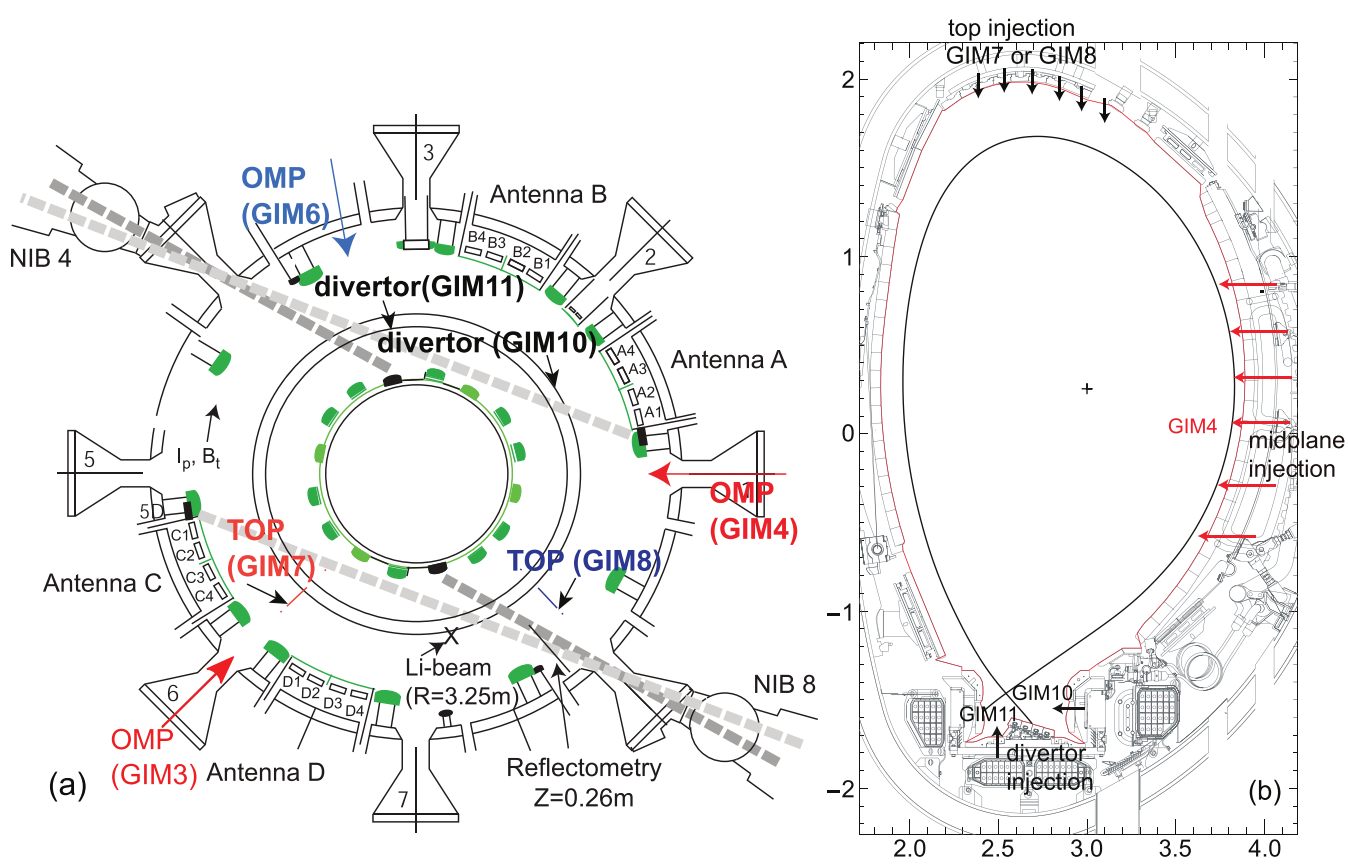

Figure 3. (a) Top view of JET illustrating the toroidal locations of the plasma facing wall components, the GIMs and the key density diagnostics. Adapted with permission from [35]. (c) 2014 Euratom. (b) Poloidal cross-section of JET shows the gas route puffed from different GIMs. Reproduced courtesy of IAEA. Figure from [5]. Copyright 2016 IAEA.

the electron temperature which again is beneficial from the point of view of RF specific heat loads and impurity release.

Besides local gas puffing, reducing the radial distance from the separatrix to the ICRF antennas is also a potential way to increase the ICRF coupling. However, this technique may significantly increase the power fluxes to the main chamber limiters (including the antenna limiters) or blanket module (in ITER). Also the minimum gaps in ITER will be constraint by the response time of the plasma control system. In comparison, gas puffing is a more flexible and efficient way to improve the ICRF coupling. Moreover, local gas puffing is able to reduce the impurity influxes from antennas, as discussed before. Despite these merits, local gas puffing can influence the diagnostics nearby.

The paper is organized as follows. In section 2, the simulation setup is presented, including the settings of the gas puff and gas pump, the plasma facing components, the computational grid and the validations of the background plasmas. In section 3 , the simulation results are discussed. These include the neutral and electron densities during different gas puffing, the calculations of the ICRF coupling resistances, the mechanisms of gas puff effects on SOL density and ICRF coupling. In section 4, the combined gas puff scenarios are investigated. In section 5, conclusions and an outlook are given.

\section{Simulation setup}

EMC3-EIRENE is a code package which self-consistently couples the Edge Monte-Carlo 3D plasma fluid code (EMC3) [18] and the kinetic neutral Monte Carlo code (EIRENE) [27]. EMC3 solves a set of time-independent Braginskiilike equations for mass, parallel momentum, electron and ion energy with anomalous transport coefficients. EIRENE is a Monte-Carlo solver of the Boltzmann equation for the neutrals. The parallel transport is treated purely neoclassically in EMC3 while the perpendicular transport coefficients $D_{\perp}$ and $\chi_{\perp}$ are specified as free parameters. These free parameters can be derived through comparisons of the simulated plasma parameters with the experimental ones. Besides its wide applications in stellarators [28, 29], EMC3-EIRENE is also attracting extensive interest for its implementation in tokamaks where some 3D SOL physics aspects need to be accounted. For example, the code was applied to model ICRF antenna coupling experiments $[6,8]$, the influences of the Resonant Magnetic Perturbation fields [30], the 3D edge plasma convection [20] and the plasma-wall interactions $[20,31]$. Note that drifts and volume recombination are not calculated in the present versions of the EMC3-EIRENE code. It is worth mentioning that progresses have been made in applying prescribed drifts in the code [20]. These prescribed drifts can be any drifts while they have to be calculated in advance and read as input fields.

Before running our simulations, it is important to first build a realistic simulation model. The layout of the JET torus is illustrated in figure 3, including the main wall structures, the gas injection modules (GIMs) and the locations of the key density measurements. A toroidal $360^{\circ}$ computation grid is built based on the EFIT equilibrium [32] from discharge \#84476 at $50.0 \mathrm{~s}$ (see figures 4 and 5). Toroidally, the computational grid is composed of sixteen equally constructed segments and each segment is divided into sixteen parts. The JET vacuum vessel is divided toroidally into eight octants, thus two segments in our simulations are used to represent one octant. A further increase of the toroidal resolution of the grid results in a noticeable increase of the Monte Carlo noise (both in EMC3 and EIRENE). In the following, $0^{\circ}$ is set at the boundaries of octant one and octant eight. In the poloidal cross-section, the grid is divided into the core, SOL and private flux region 


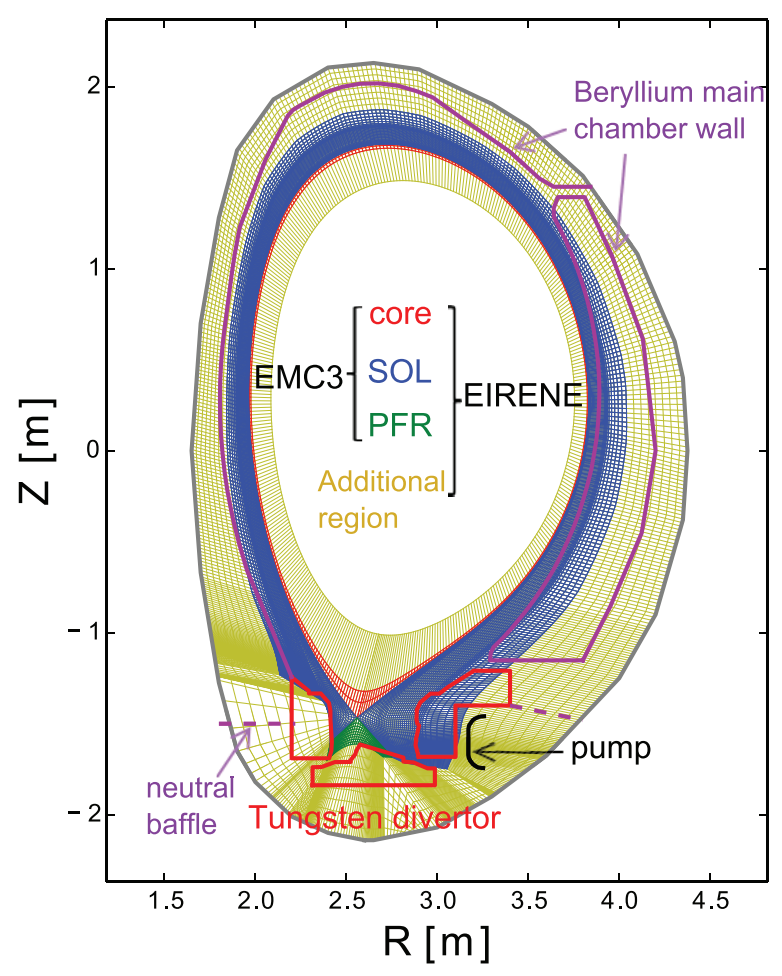

Figure 4. A poloidal cross-section of the EMC3-EIRENE computation grid.

(PFR). In the SOL the grid resolution is $39 \times 481 \times 256$ (radial $\times$ poloidal $\times$ toroidal) cells. A relative high resolution is specified in the regions with physics of interest: the divertor and the outer mid-plane regions in front of the limiters. In addition to the EMC3 grid, the EIRENE grid includes an 'additional region' (figure 4, in yellow color) bounded by the vessel wall so that the neutral transport inside the whole vessel can be calculated. A toroidally symmetric gas pump (cryo pump) is set at its actual position. This pump is the dominant gas pump and the contributions of other gas pumps (such as turbo pump) are negligible for the experiments investigated. The reflection coefficient of the pump surface is set as 0.98 , meaning that $2 \%$ of the neutrals hitting the pumping surface are absorbed while the rest is reflected. With this setting, the gas pump rate is equal to the gas puff rate $\left(1.7 \times 10^{22}\right.$ electrons $\mathrm{s}^{-1}$ ) and the electron density matches the experimental one in our simulations. Two neutral baffles (the purple dashed lines in figure 4) are set in regions outside the divertor. This is to make the neutral transport in those regions as realistic as possible. In reality hardly any gas can pass through the locations of the neutral baffles.

In steady state JET H-mode plasmas, the recycling fluxes from the divertor and main chamber wall are the primary particle sources. In particular, the limiters in the low field side have strong interactions with the plasma. They play a crucial role in confining the plasma and generating recycling fluxes. Thus, all these limiters including eight Wide Poloidal Limiters (WPLs), two ITER-Like-Antenna (ILA) limiters and one Narrow Poloidal Limiter (NPL) are taken into account in our simulations (see figure 5). The different types of limiters have different widths and different radial

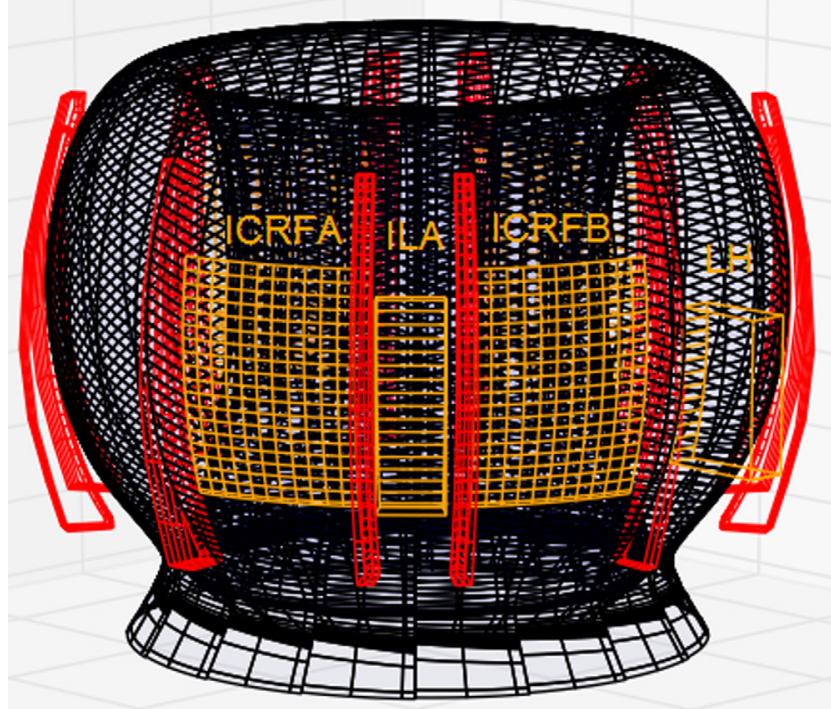

Figure 5. 3D structure of the computational grid, the limiters and the antennas.

positions. As a result they will induce toroidal inhomogeneities in the SOL. This will be discussed later in more detail. The toroidal axisymmetric plasma facing components in our model include the inner and outer targets and the roof baffle. Because the inner limiters (those on the high field side) are identical and axisymmetric, the inner wall is placed at the same location as the leading edges of these inner limiters. For the wall materials, tungsten (W) and Beryllium (Be) corresponding to the divertor and main chamber wall respectively, are used in our simulations. No sputtering is included in the simulations, the use of different wall materials affects the reflection model in EIRENE.

In our simulations, the GIMs are either set at the realistic positions or set in such a way that the simulated gas is equivalent to the experimental one (in terms of the spreading and intensity of the gas). For the top gas puff (GIM7 or GIM8), eight poloidally evenly distributed point sources in the top of the vessel are used to inject the same amount of gas as in the experiment. We have used eight point sources so that the poloidal distribution of the gas is homogeneous enough. For the divertor gas puff (GIM11), eight toroidally evenly distributed point sources have been set at the realistic divertor positions to generate the experimental equivalent gas. The settings of the mid-plane GIMs (GIM3, GIM4 or GIM6) are more complicated. This is because the midplane GIMs are located very deep in the so called A-port. They are far away from the main chamber and are outside our simulation domain. As the gas is transported radially inward from the GIM, it keeps spreading poloidally and toroidally filling essentially the whole A-port. A rather homogenous gas is found at the cross-section where the A-port is connected to the main chamber. To simulate this inside the vessel, we have put five point sources distributed equidistantly in poloidal direction $(\sim 50 \mathrm{~cm}$ away from the seperatrix) at the outer mid-plane to simulate the experimental gas puff as realistically as possible. Before the gas reaches the hot plasma and is ionized, it has already spread 


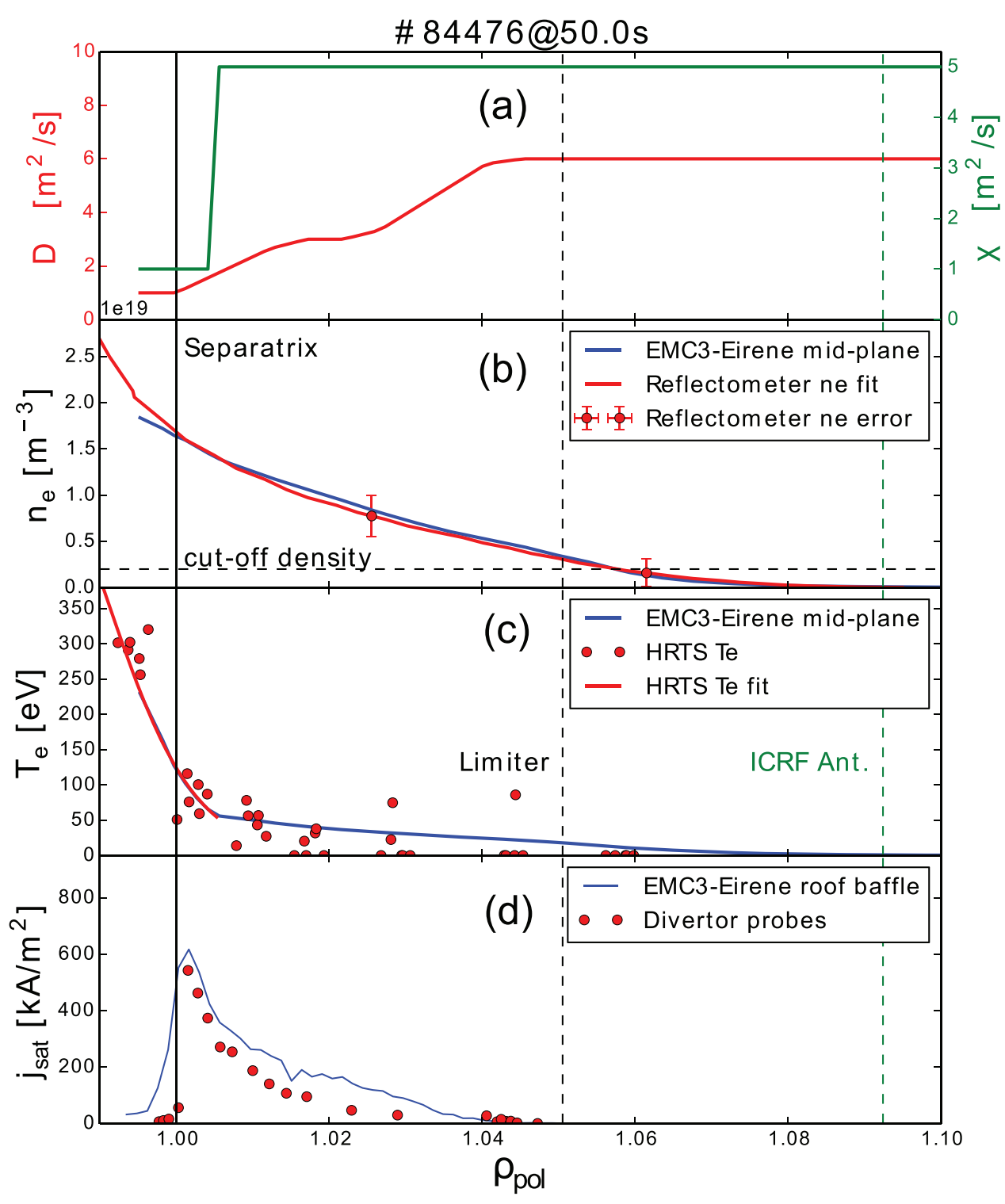

Figure 6. Matching the background plasma parameters with experiments: (a) perpendicular plasma transport coefficients $D_{\perp}$ and $\chi_{\perp}$; (b) comparisons of mid-plane electron density, the error bars correspond to the statistical dispersion of the reflectometry data; $(c)$ comparisons of mid-plane electron temperature; $(d)$ comparisons of particle fluxes to the divertor (around the outer strike point in the roof baffle).

to a rather large extent. At the leading edge of the limiters, the spreading of the gas is in the range of $(-0.85 \mathrm{~m}, 0.85$ $\mathrm{m})$ poloidally and about ten degrees toroidally (depending on the wall structures nearby). These features are well captured in our simulations. The setup procedure of the JET mid-plane GIMs in our simulations is similar to the one implemented in AUG (see [8]) but more sophisticated. In AUG only one mid-plane GIM is needed to generate the experimental equivalent gas.

The simulation strategy is as follows. First, a reference pulse with symmetrical divertor gas puffing is simulated, and in particular transport parameters are adjusted to match the measured profiles of $n_{\mathrm{e}}, T_{\mathrm{e}}$ and divertor saturation current. This validated plasma is then assumed as background plasma, and it is used in the simulations to study the changes in the SOL when puffing gas from other locations, for example at specific toroidal locations at the outer mid-plane or at the top of the vessel.
Our divertor reference is the JET pulse 84476 where the divertor GIM11 was used. The separatrix averaged density is $2 \times 10^{19} \mathrm{~m}^{-3}$ and the net input power is $14 \mathrm{MW}, P_{\text {net }}=P_{\mathrm{NBI}}$ $+P_{\mathrm{ICRH}}+P_{\mathrm{OH}}-P_{\mathrm{rad}}$. These two parameters are derived from experimental measurement and they are regarded as inner boundary conditions. Note that experimentally these parameters remained unchanged when further using top or mid-plane gas puff. Because no impurities are considered in our simulations, the power radiated by impurities has been subtracted from the total heating power to evaluate the net power. For the discharges considered in our studies, $B_{\mathrm{T}}=2.7 \mathrm{~T}, I_{\mathrm{p}}=2.5$ MA, $P_{\text {rad }}=6 \mathrm{MW}$ and the minimum distance from the separatrix to the wall is $6 \mathrm{~cm}$. To correctly describe the cross-field transport of the plasma, the upstream and downstream profiles in our simulations are best matched to experiments by adjusting the perpendicular plasma transport parameters (i.e. the so-called 'free parameters' mentioned above). The results of this procedure are shown in figure 6 . The upstream profiles 
Table 1. The simulated cases in parallel with the experiments.

\begin{tabular}{lllll}
\hline Simulated cases & & $\begin{array}{l}\text { Gas valve positions } \\
\text { (toroidal) }\end{array}$ & Corresponding experiments & Gas puff rate \\
\hline Divertor gas puff & GIM11 & $\begin{array}{l}\text { Toroidally evenly } \\
\text { distributed }\end{array}$ & $\# 84476, \# 84485, \# 90140$ & $1.7 \times 10^{22} \mathrm{electrons} \mathrm{s}^{-1}$ \\
\hline Top gas puff & GIM7 & $\phi=-137.5^{\circ}$ & $\# 84739$ \\
& GIM8 & $\phi=-45^{\circ}$ & $\# 84740$ \\
\hline Mid-plane gas puff & GIM3 & $\phi=-135^{\circ}$ & $\# 90131, \# 90133, \# 90136$, \\
& GIM4 & $\phi=0^{\circ}$ & $\# 90137$ \\
& GIM6 & $\phi=108^{\circ}$ & $\# 84478, \# 90128, \# 90129$, \\
& & $\# 90130$ \\
\hline
\end{tabular}

include the mid-plane electron density $n_{\mathrm{e}}$ and the mid-plane electron temperature $T_{\mathrm{e}}$. The downstream profiles include the particle flux to the divertor targets $j_{\text {sat }}$ (the outer strike point is located on the right bottom of the divertor). The perpendicular transport parameters include the particle and heat diffusion coefficients $D_{\perp}$ and $\chi_{\perp}$, in which $\chi_{\mathrm{i} \perp}=\chi_{\mathrm{e} \perp}=\chi_{\perp}$. For the experimental data, we have selected the values during the full inter-ELM periods. The averaged mid-plane $n_{\mathrm{e}}$ is obtained with the following procedures: the core and pedestal density are derived from High resolution Thomson Scattering (HRTS), and the SOL profiles are derived from the reflectometer (more than 2700 reflectometer profiles are used in this averaging procedure). The density data are averaged over the time $49.5-50.5 \mathrm{~s}$. The mid-plane $T_{\mathrm{e}}$ is averaged from the HRTS data and is only reliable in the plasma core and in the near SOL. In the far SOL, the measured $T_{\mathrm{e}}$ with HRTS has large error bars. The particle fluxes to the divertor targets $j_{\mathrm{sat}}$ are measured with Langmuir probes. $j_{\text {sat }}$ is equal to the current (i.e. the particle stream) in a flux tube divided by the area perpendicular to that flux tube.

\section{Simulation results}

Once the background plasma is defined, the gas source is switched from the divertor to the main chamber (top or midplane) of the machine in our simulations. All other parameters, including the total gas puff rate $1.7 \times 10^{22}$ electrons $\mathrm{s}^{-1}$, are kept unchanged. The JET pulses studies using this procedure are listed in table 1.

\subsection{Gas puff effects on the edge plasma}

After running the simulations for all the cases, the simulated neutral and electron densities during gas puffing in the divertor, top and mid-plane are shown in figure 7. $n_{\mathrm{D} 2}, n_{\mathrm{D}}$ and $n_{\mathrm{e}}$ represent the deuterium molecular density, the deuterium atom density and the electron density, respectively. In each case, the poloidal cross-section is taken at the toroidal position where the GIM is located. According to our simulation results (figure 7), a neutral density cloud localized in front of the top or mid-plane GIMs develops during the corresponding gas puff. As the gas cloud interacts with the plasma, it is ionized locally when the electron temperature is larger than the threshold ionization temperature $(\sim 2 \mathrm{eV})$. The neutral ionization rate depends nonlinearly on the electron temperature [33]. The complex ionization processes are calculated in EIRENE based on the AMJUEL/HYDHEL database and an electron density cloud is formed in front of the GIMs where the ionization happens. The cloud of enhanced density can be seen in figure 7 (right column). The extension of the density cloud mostly depends on the spreading of the neutral gas while the peak density largely depends on the ionization rate. In the density cloud, the electron temperature is lower than the background plasma temperature. Due of the rapid motion of charged particles along field lines (i.e. the parallel transport), density in regions which are magnetically connected to the density cloud are soon enhanced. The density increase in the SOL depends on the structures of the field lines, on the density cloud as well as on the perpendicular transport. If these field lines spread widely, the high density source is shared by a large region so that the increase of density is global but not substantial; if the field lines remain close to each other over a long distance, the density increase can be significant but more local.

Comparisons of the simulated gas pressure and the experimental one are made for the mid-plane gas puff case (GIM4) (figure 8). It is shown that the maximum value of the gas pressure (gas pressure at the toroidal position of the GIM) and the toroidal distributions of the gas pressure are well reproduced in our simulations. The local gas pressure increases (the small peaks) are due to the local recycling at the limiters. The quantitative agreements of the gas pressure confirm that the settings of the mid-plane GIMs in our simulations are reasonable.

Furthermore, we have compared the experimental and simulated electron density for the divertor (GIM11) and mid-plane (GIM4) gas puff cases (figure 9). The comparisons are made at the reflectometer position, i.e. toroidally at $\phi=-52.5^{\circ}$ and vertically at the plasma outer mid-plane. Only the reflectometer data during the inter-ELMs phases is used. Quantitative agreement is found between the simulated and experimental density profiles. A match within $95 \%$ is seen during divertor gas puffing. Small differences in the limiter shadow and in the near SOL are observed during mid-plane gas puffing. Nevertheless these differences are still within the experimental error bars. This good agreement further confirms that 

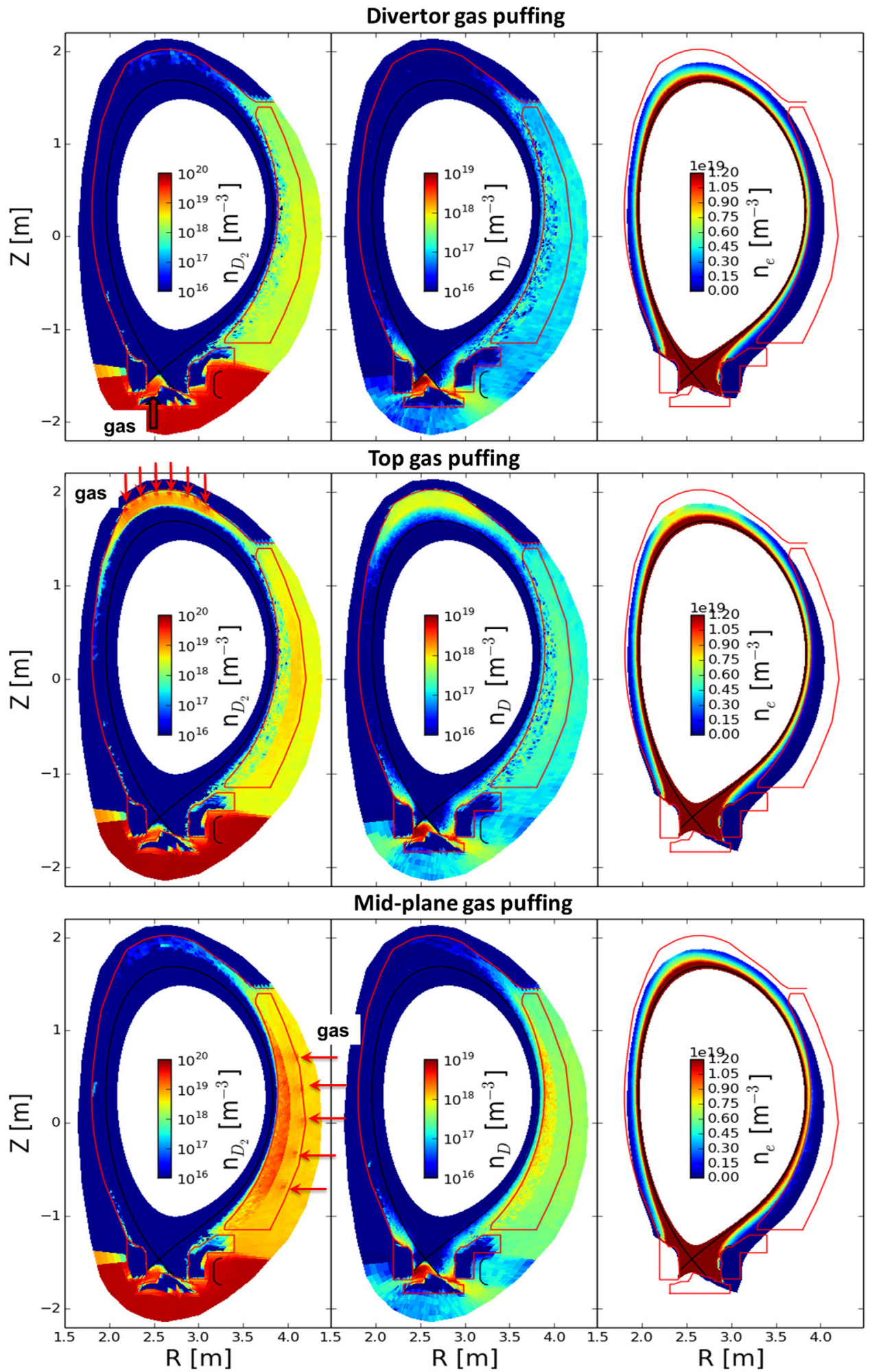

Figure 7. Poloidal cross-sections of the neutral density ( $n_{\mathrm{D} 2}$ for molecular and $n_{\mathrm{D}}$ for atom) and electron density $\left(n_{\mathrm{e}}\right)$ during divertor, top and mid-plane gas puffing, respectively. The poloidal cross-sections are chosen at toroidal positions where the GIMs are located.

the settings of the mid-plane GIMs in our simulations are reasonable, and gives us confidence that our simulations are able to reproduce the experiments at the quantitative level.

To understand the toroidal density distribution, toroidal cross-sections of the density profile at the outer mid-plane $(z=0.3 \mathrm{~m})$ are shown in figure 10 for the divertor (GIM11), top (GIM7) and mid-plane (GIM4) gas puff cases. In this figure the toroidal positions of the ICRF antennas (in green color) and GIMs (white dashed lines) are also indicated. The orange line represents the position of the fast wave cut-off density during 


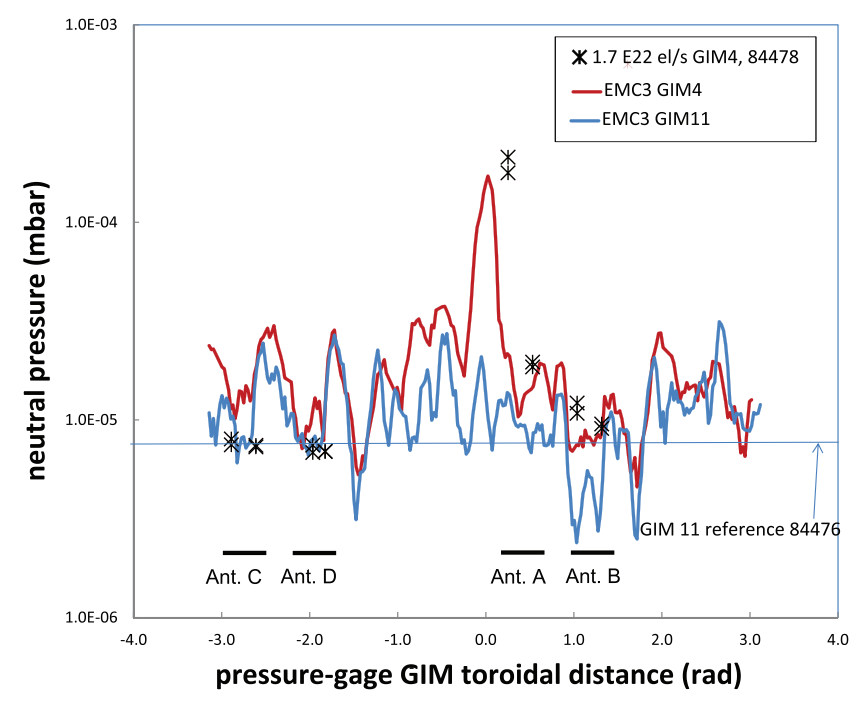

Figure 8. Comparisons of simulated and experimental gas pressure during mid-plane (GIM4) and divertor (GIM11) gas puffing. The pressure measurements are made in the $\mathrm{A} 2$ antennas in-vacuum transmission lines (un-pumped).

divertor gas puffing and is used as reference. The red line is the position of the fast wave cut-off density during top or mid-plane gas puffing for dipole (out-of-phase) phasing operation.

It can be seen from the simulations that during divertor gas puffing (GIM11), the electron density in the near SOL is quite uniform while a toroidal inhomogenous density is found in the far SOL. This is due to the effects of the limiters. For instance the limiters near antennas A and B are NPL and ILA limiters. They are narrower and radially further retracted than the WPLs. Consequently the density in front of antennas A and $\mathrm{B}$ is higher than the ones in other toroidal positions.

Compared to the divertor gas puff, the top gas puff (GIM7) increases the density almost uniformly in the toroidal direction but to a small extent. The density increase in front of antennas A and B is slightly larger than that in front of antennas $\mathrm{C}$ and $\mathrm{D}$, which is again due to the effects of the limiters. While changing the top gas puff locations, for instance when switching from GIM7 to GIM8, the SOL density increase remains the same. This suggests that the density increase during top gas puffing is independent from the toroidal positions of the top GIMs. During mid-plane gas puffing, the density increase in the SOL is significantly larger but very local. The largest density increase is found in regions close to the outer mid-plane GIMs. This large density increase gradually vanishes as one moves away toroidally from the GIM. These findings are consistent with the findings in AUG [8] and JET-ILW [5] experiments.

To have a more accurate description of the density increase in front of the antennas, we have compared the average density in front of the antennas for all the gas puffing scenarios (figure 11). The density is averaged on the flux surfaces and then mapped to the mid-plane. It is calculated with the formula

$$
\left\langle n_{\mathrm{e}}(\psi)\right\rangle=\frac{1}{\Delta \theta \Delta \Phi} \int_{\theta_{0}-\Delta \theta / 2}^{\theta_{0}+\Delta \theta / 2} \int_{\Phi_{0}-\Delta \Phi / 2}^{\Phi_{0}+\Delta \Phi / 2} n_{\mathrm{e}}(\psi, \theta, \Phi) \mathrm{d} \theta \mathrm{d} \Phi,
$$

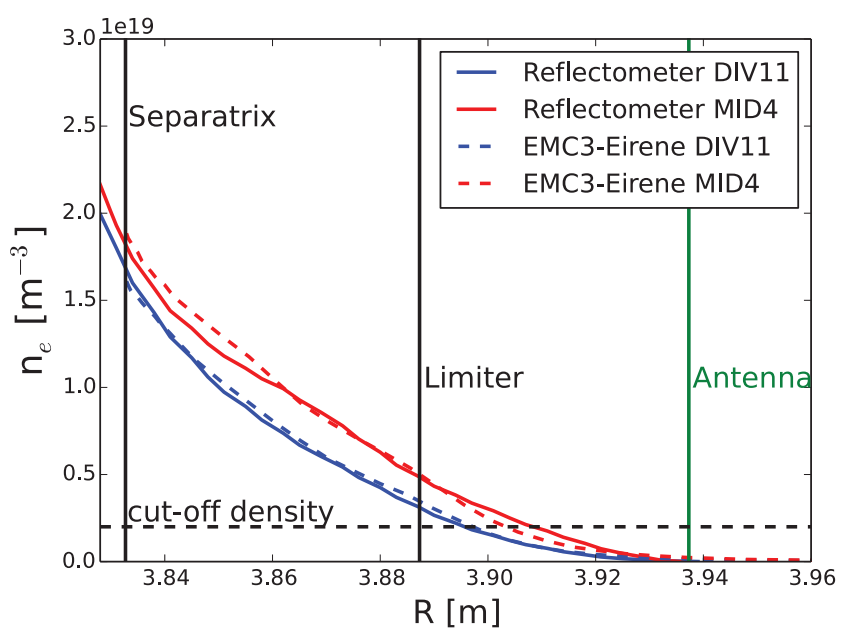

Figure 9. Comparisons of simulated and experimental density (measured with reflectometer) for mid-plane (GIM4) and divertor (GIM11) gas puffing. DIV11 means divertor puffing with GIM11 and MID4 means mid-plane puffing with GIM4

in which $\theta_{0}$ and $\Phi_{0}$ represent the poloidal and toroidal angles of the antenna center, $\Delta \theta$ and $\Delta \Phi$ are the poloidal and toroidal angle extensions of the antenna, respectively. $\left\langle n_{\mathrm{e}}(\psi)\right\rangle$ is then transformed to $\left\langle n_{\mathrm{e}}(R)\right\rangle$ at the mid-plane $(Z=0.3 \mathrm{~m})$. Our results indicate that the mid-plane GIMs are most effective in increasing the density for the antennas nearby. For instance GIM4 is nearest to antenna A and the largest density increase is found in front of antenna A. GIM3 is located in the middle of antennas $\mathrm{C}$ and $\mathrm{D}$, thus the density in front of these two antennas increase substantially. The top gas puff (GIM7) increases the density for all the antennas to an almost similar but small level.

In JET, the distance from the leading edge of the limiter to the antenna strap is about $5 \mathrm{~cm}$. Thus, even though the position of the cut-off density is behind the leading edge of the limiters during divertor gas puffing, there is still a considerable distance between the position of the cut-off density and the antenna straps. The main chamber gas puff can change the density gradient and the evanescent distance in the limiter shadow, which will lead to an increase of the ICRF power coupling.

The field line tracings starting from the top and mid-plane gas clouds to the ICRF antennas are shown in figure 12. Field lines starting from the top toward the mid-plane spread widely. This explains the evenly distributed toroidal density increase during top gas puffing. In contrast, field lines starting from the mid-plane toward the top are much more concentrated. This is the reason for the significant but localized density increase during mid-plane gas puffing. Although field line tracing is useful for a first hint for the regions of enhanced density, it is far from enough. This is because other factors mentioned above are not taken into account, such as the spreading of the neutral gas, the ionization and the complicated field line structures in the SOL. In ITER, the GIMs are located in the outer top of the vessel and are far away from the main chamber. The gas has to go through the gaps between the wall modules to 


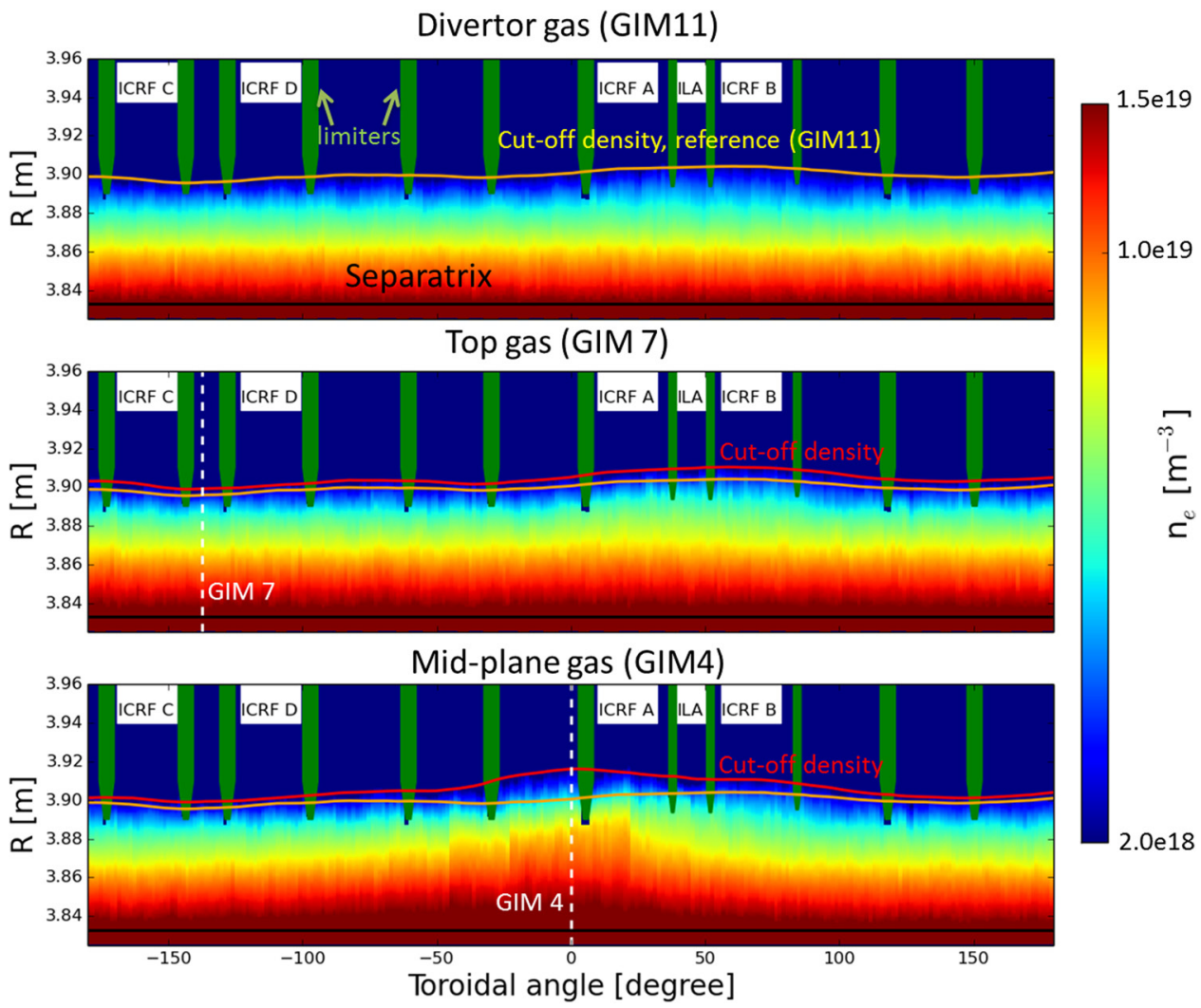

Figure 10. Toroidal cross-sections of the electron density in the outer mid-plane for the divertor, top and mid-plane gas puff cases. The yellow solid line is the position of cut-off density for divertor gas puffing and reported in the second and third subfigures as reference line. The red solid-line is the position of cut-off density for top or mid-plane gas puffing.

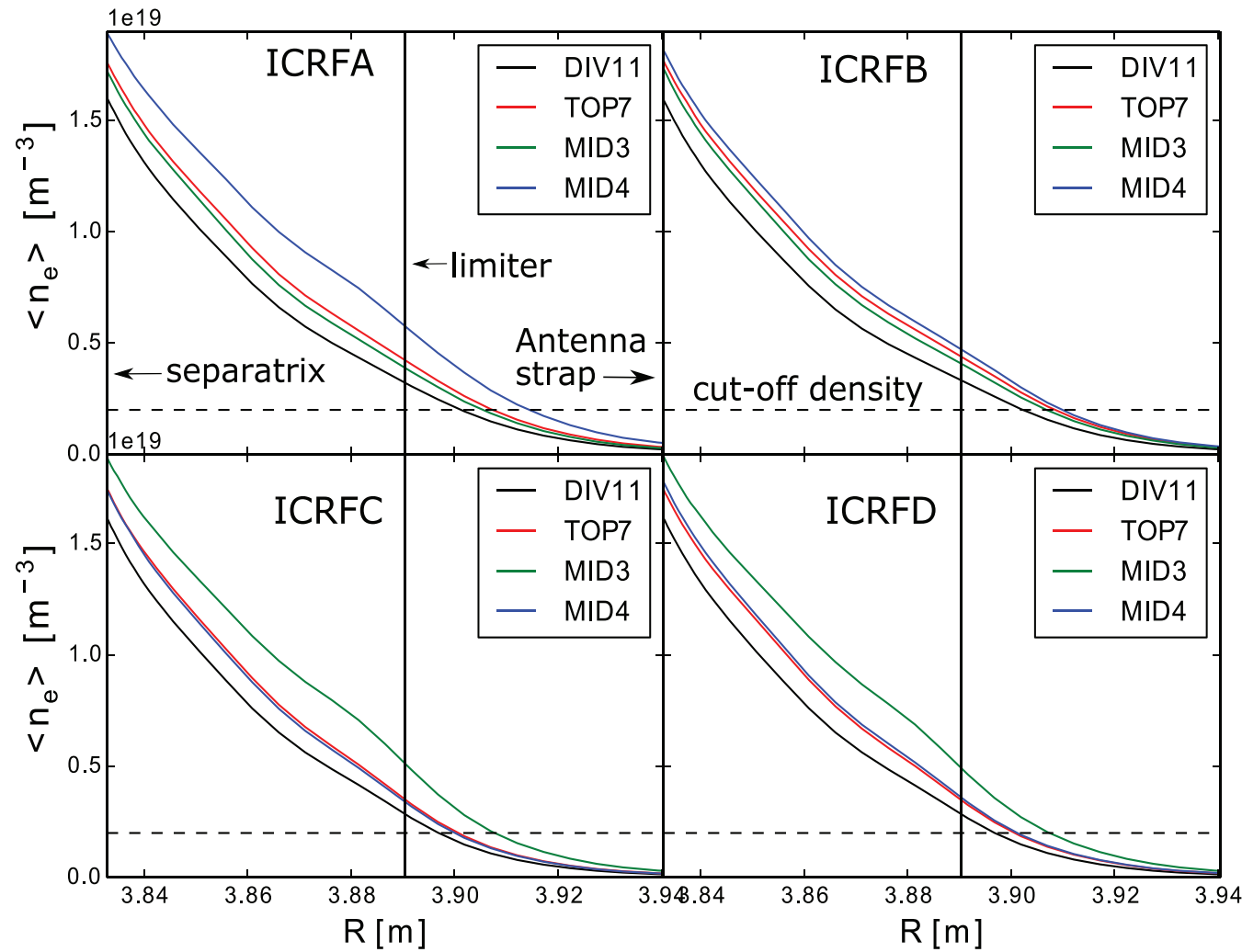

Figure 11. The calculated average density in front of the ICRF antennas for the divertor (GIM11), top (GIM7) and mid-plane (GIM3 and GIM4) gas puff cases. ICRFA means ICRF antenna A. 


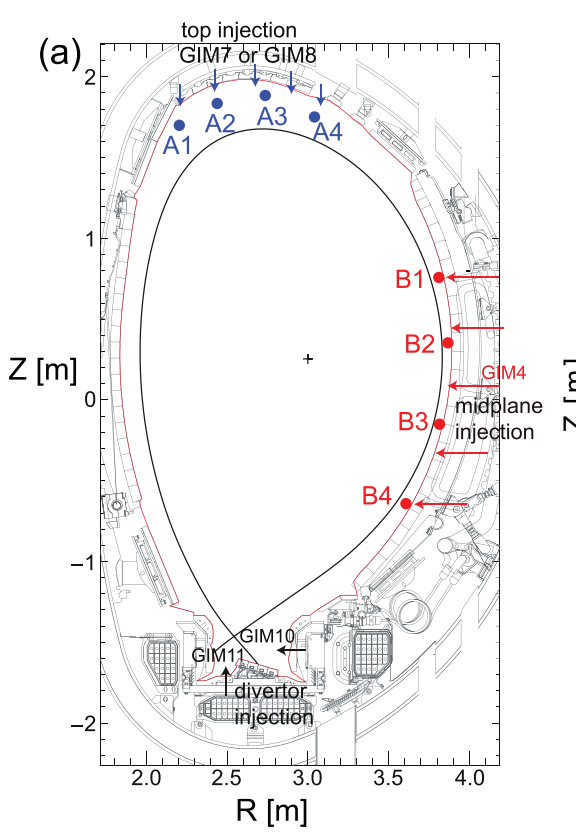

(b)

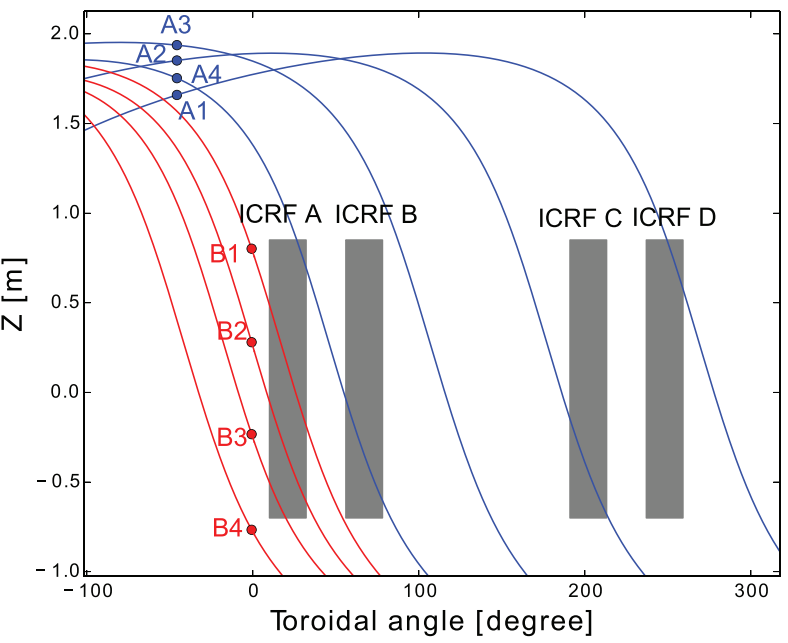

Figure 12. Field line tracings starting from the top gas cloud (points $\mathrm{A} 1, \mathrm{~A} 2, \mathrm{~A} 3$ and $\mathrm{A} 4$, toroidal angle $=-45^{\circ}$ ) and mid-plane gas cloud (points B1, B2, B3 and B4, toroidal angle $=0^{\circ}$ ), respectively.
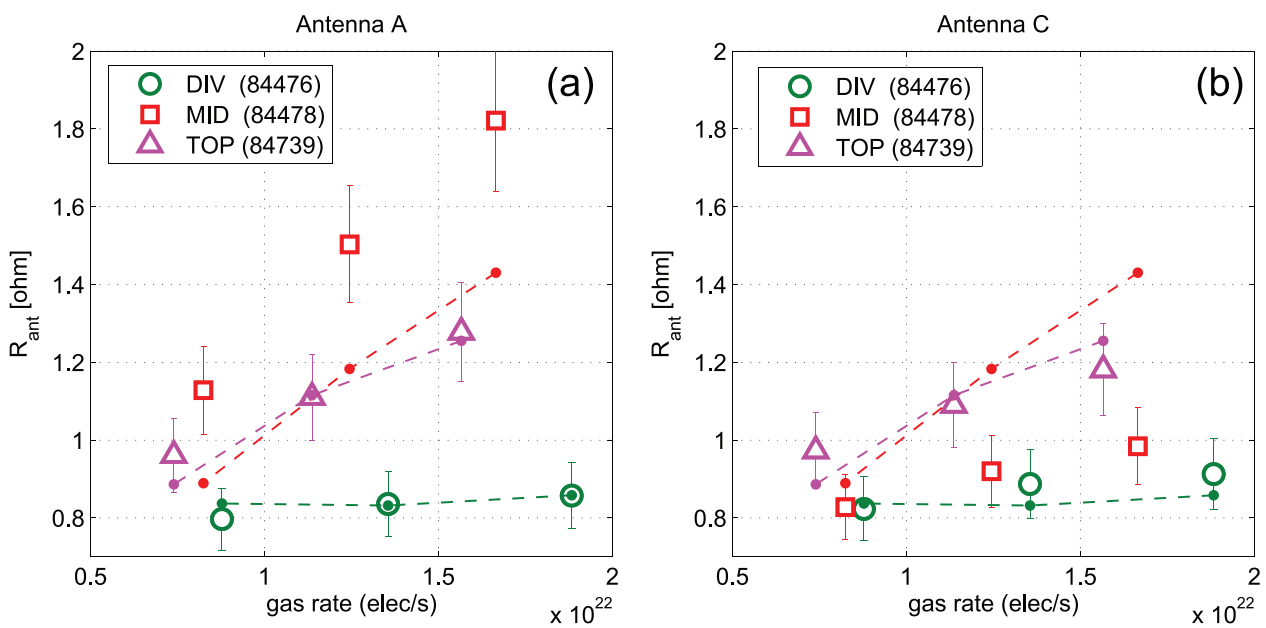

Figure 13. Comparison between the measured coupling resistances for $(a)$ antenna $\mathrm{A}$ and $(b)$ antenna $\mathrm{C}$ and the expected coupling resistances (dashed lines) computed with the 1D slab RF code using the experimental SOL density profiles measured with the interferometer. The free parameter in the 1D RF coupling code is adjusted to match the experimental coupling resistance of antenna A in pulse 84476 (divertor injection) at maximum gas rate.

reach the plasma. To have a correct prediction for the density at the ICRF antenna, comprehensive EMC3-EIRENE simulations that take into account the $3 \mathrm{D}$ equilibrium and an accurate description of the wall components and of the geometry/ location of the various GIMs are necessary.

\subsection{Gas puff effects on ICRF coupling}

As discussed in the introduction, the ICRF coupling depends critically on the SOL density in front of the antennas. Different gas puffing methods can result in different SOL density and hence different ICRF coupling. To understand the impact of the density modifications when using different gas puffing scenarios on the ICRF coupling, a simple 1D fast wave RF coupling code [10] was used. The code computes the 1D RF electric fields for a given density profile using Stix's cold dielectric tensor for the fast wave [3] and the coupling resistance is estimated by calculating the poloidal averaged Poynting flux integral at the plasma edge $P_{\mathrm{RF}}$ (i.e. the coupled RF power) for a given current density $I_{\mathrm{A}}$ at the antenna, i.e. $R_{\mathrm{c}}=2 P_{\mathrm{RF}} / I_{\mathrm{A}}^{2}$. Because of its oversimplified geometry (infinitely poloidally long straps, uniform current density, no feeders, no slow waves, etc), this code does not provide accurate values of the RF coupling resistance itself, but the relative coupling changes due to the SOL density modification are believed to be satisfactorily captured.

A benchmark/validation of the code is shown in figure 13. One sees that once the $1 \mathrm{D}$ RF coupling code has 

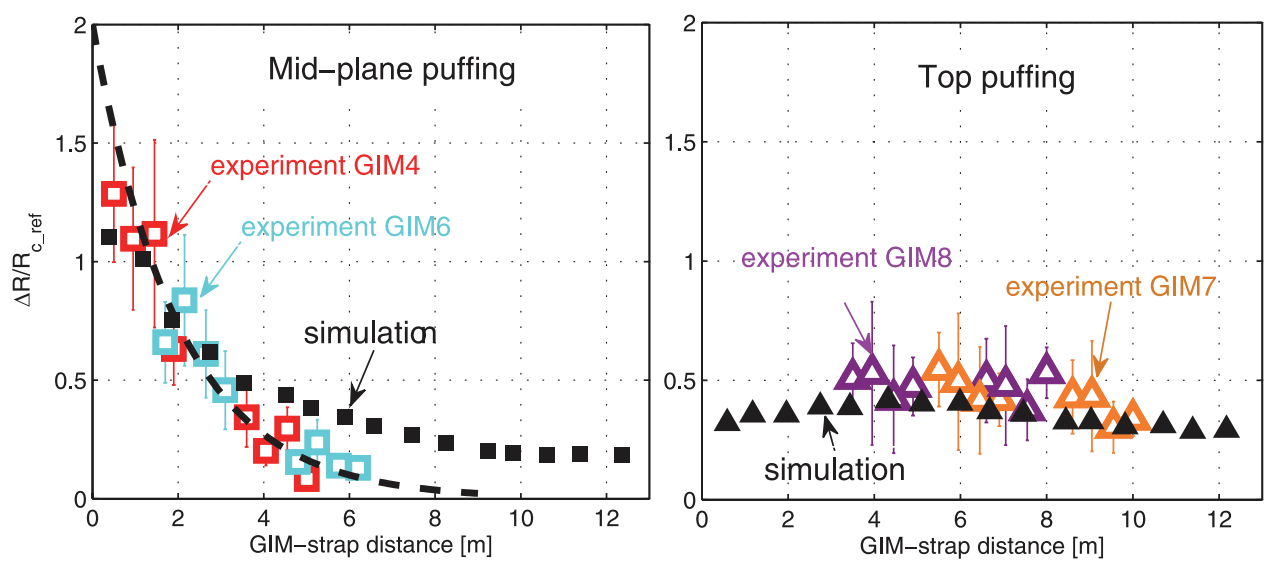

Figure 14. Relative change of coupling resistances (RCCR) versus GIM-antenna strap distance. For the calculated RCCR values, because they are largely symmetry along phi $=0^{\circ}$, only those in the toroidal range $\left[0,180^{\circ}\right]$ are shown in the figure. The experimental RCCR values are reproduced from [10]. Left: RCCR during mid-plane gas puffing, in which the dashed line is a fit of the experimental data. Right: RCCR during top gas puffing. Reprinted from [10], Copyright 2015, with permission from Elsevier.
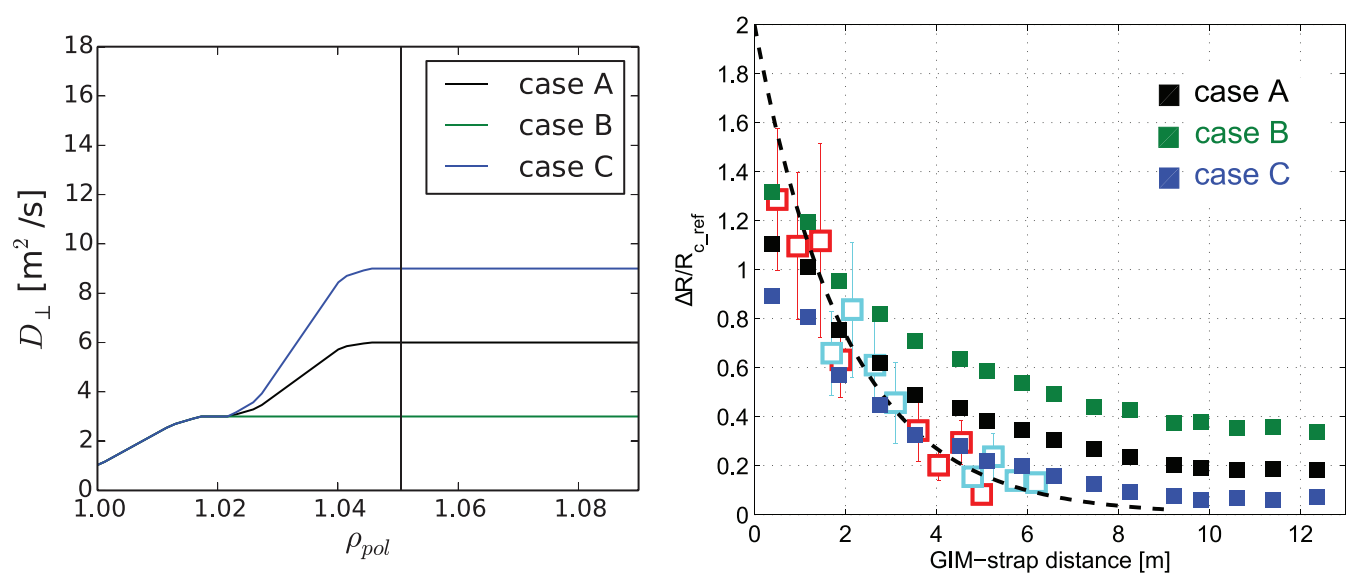

Figure 15. Influences of the particle transport coefficient $D_{\perp}$ on the ICRF coupling. Left: particle transport coefficients in the three cases investigated; right: the corresponding relative change of coupling resistances.

been calibrated, the coupling resistances predicted for both antennas with divertor (DIV) and TOP gas injection are close to the respective experimental values, indicating that the 1D coupling code is capturing the main RF physics in the process. The coupling resistances are calculated based on the SOL profiles measured by the interferometer and therefore reproduce well the cases where the density modification due to gas injection is toroidally homogeneous. For MID-plane gas injection close to antenna A (\#84478, GIM4), one sees that the predicted coupling is underestimated for antenna A and overestimated for antenna $\mathrm{C}$ (which is far away). This is because the SOL density perturbation is toroidally localized in the case of MID-plane fuelling and the interferometer (which is located in between antenna A and antenna C) registers a smaller SOL density than the one present in front of antenna A (close to the gas injection point) but still larger than the one seen by antenna $\mathrm{C}$ (whose coupling resistance is almost insensitive to the mid-plane gas injection in this case).

The 3D densities from the EMC3-EIRENE simulations were poloidally averaged within the range $\left[Z_{\min }, Z_{\max }\right]=[-0.6 \mathrm{~m}$, $0.6 \mathrm{~m}]$. The 1D density profiles in front of each antenna strap (at a given toroidal position) were used in the coupling calculations. After obtaining the $R_{\mathrm{c}}$ values from the $1 \mathrm{D}$ coupling code for different toroidal positions and various gas puffing cases, the relative change of coupling resistance (RCCR) is then calculated with $\frac{R_{\mathrm{c}}-R_{\mathrm{c} 0}}{R_{\mathrm{c} 0}}$ (i.e. $\frac{\Delta R_{\mathrm{c}}}{R_{\mathrm{c} \_ \text {ref }}}$, where $R_{\mathrm{c} \_ \text {ref }}=R_{\mathrm{c} 0}$ and $\left.\Delta R_{\mathrm{c}}=R_{\mathrm{c}}-R_{\mathrm{c} 0}\right)$. Here $R_{\mathrm{c} 0}$ is the coupling resistance during divertor gas puffing and used as a reference value. Good agreement is found between the simulated and experimental RCCR values (figure 14). In the experiment, $R_{\mathrm{c}}$ can only be measured at the toroidal positions of the antennas. In our simulations, we have used 32 density profiles with toroidal distance $\sim 0.7 \mathrm{~m}$ between each other (some of these toroidal positions are a little shifted to avoid the positions of the limiters) for the $R_{\mathrm{c}}$ calculations of an hypothetical antenna at these toroidal positions. This gives how RCCR varies with the toroidal position. The simulations (figure 14) indicate that during top gas puffing, $R_{\mathrm{c}}$ is increased everywhere by an almost same moderate value (by $\sim 40 \%$ ). The small variations of the RCCR values in the toroidal direction are due to the effects of the limiters (see figure 10). During mid-plane gas puffing, $R_{\mathrm{c}}$ is increased most significantly for antennas near the GIMs (as much as by $130 \%$ in experiments). It decays exponentially as the GIMstrap distance increases. Quantitative agreement is found between the experiments and simulations when the GIM-strap 


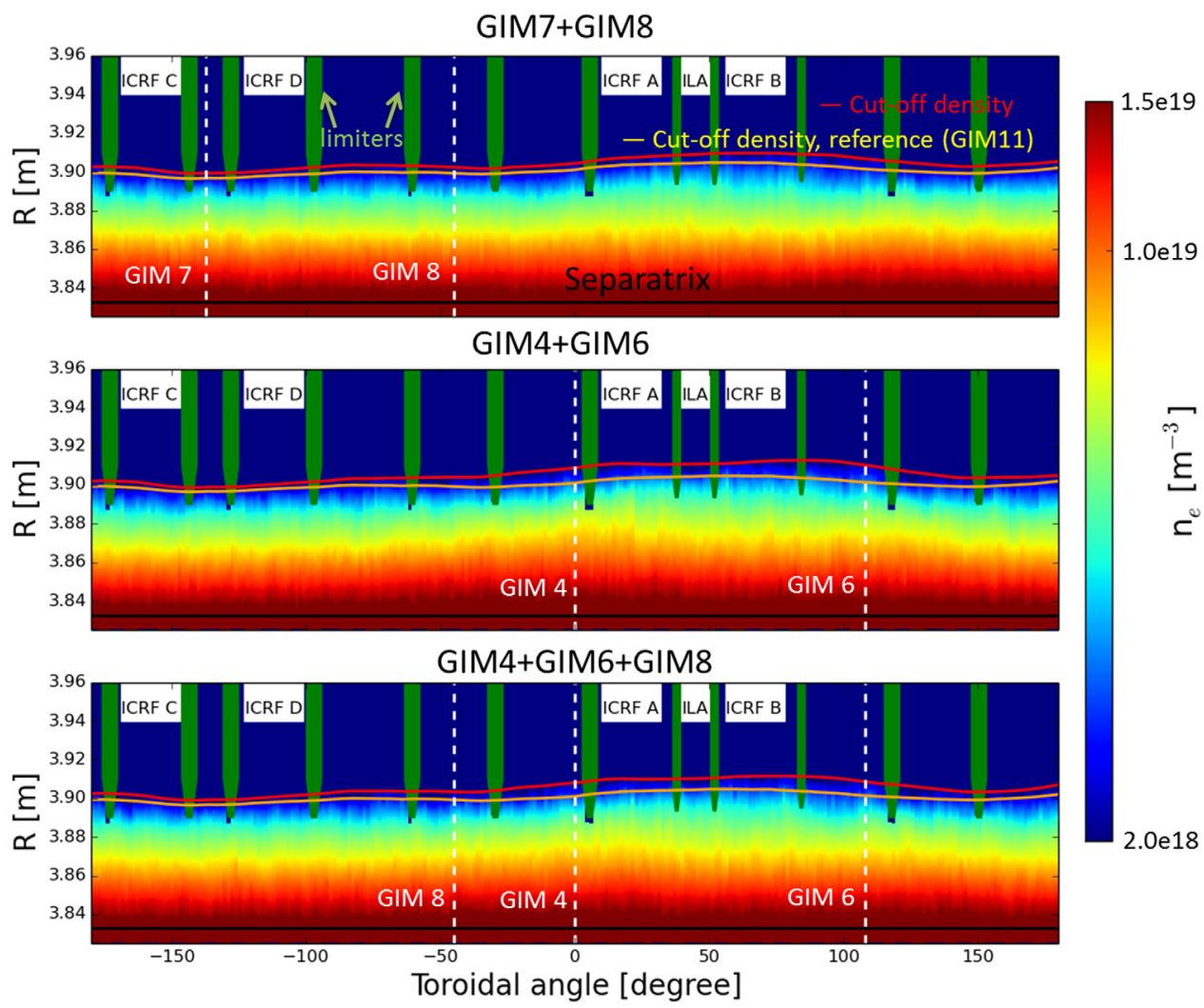

Figure 16. Toroidal cross-sections of the electron density in the mid-plane for combined gas puffing scenarios.

distance is smaller than $3 \mathrm{~m}$. However, the simulated RCCR curve decays more slowly than the experimental one. Many reasons can be responsible for this: 1 . no drift is included in the EMC3-EIRENE code; 2. the simplified antenna geometry and reduced physics in the $1 \mathrm{D}$ antenna code; 3 . the errors in the experimental data which is used for validating the background plasma (section 2); 4. errors of the experimental $R_{\mathrm{c}}$ values; 5 . the simplified wall structures in the EMC3 simulations; 6 . the imprecise settings of the transport parameters in our simulations; 7. local ionization effects due to the large RF fields close to the antennas that are not included in the simulations [22]; 8. radiation patterns from impurities and plasma; 9. plasma-wall interaction (sputtering, reflection on metallic wall versus carbon wall).

To understand the influence of the transport parameters on the RCCR values, further EMC3-EIRENE simulations are carried out with different particle transport parameters specified in the far SOL (case A: $D_{\perp}=6 \mathrm{~m}^{2} \mathrm{~s}^{-1}$; case B: $D_{\perp}=3 \mathrm{~m}^{2} \mathrm{~s}^{-1}$; case C: $D_{\perp}=9 \mathrm{~m}^{2} \mathrm{~s}^{-1}$ ). In each case the divertor (GIM11), top (GIM7) and mid-plane (GIM4) gas puff scenarios are investigated. Note that case A is the same as that in sections 3 and is used as a reference. The results (figure 15) indicate that compared to case A, a lower $D_{\perp}$ in the far SOL (case B) results in a higher SOL integrated density and an upward shift of the RCCR curve, while a higher $D_{\perp}$ in the far SOL (case C) results in a lower SOL integrated density and a downward shift of the RCCR curve. The RCCR decay lengths in these cases are almost the same while the magnitudes of the RCCR values are different. This indicates that the $D_{\perp}$ values can have an impact on the magnitude of the RCCR values with higher local density increase when perpendicular transport is reduced, but not on the toroidal decay length of the SOL density.

\section{Further studies}

The top or mid-plane gas puff scenarios described previously are all with local gas using a single GIM. Because the RF antennas are toroidally distributed in JET-ILW and AUG, the maximum RF power capability is obtained when using distributed main-chamber gas fueling to homogeneously enhance the plasma density in front of the antenna and thus the coupling resistance of all antennas. This technique has proved very effective in JET-ILW [34] but some unexpected effects have been observed: The coupling enhancement obtained with combined mid-plane and top fueling is stronger than what would be predicted by summing the individual contributions for giving the same total injection rate. To further interpret these observations, in this section the combined gas puff method-puffing with several main chamber GIMs simultaneously-are investigated. The following cases are simulated: I. Two top GIMs, GIM7 (50\%) + GIM8 (50\%); II. Two mid-plane GIMs, GIM4 (50\%) + GIM6 (50\%); III. Two mid-plane GIMs and one top GIM, GIM4 (33\%) + GIM6 $(33 \%)+$ GIM8 $(33 \%)$. The total gas puff rate in all these cases is equal to $1.7 \times 10^{22}$ electrons $\mathrm{s}^{-1}$. The gas puff rate of each GIM is shown as percentage of the total one.

Similar to section 3, the toroidal cross-sections of the density and the averaged density in front of the antennas are shown in figures 16 and 17, respectively. It is shown that the density increase with two top GIMs (GIM7 + GIM8) is similar to 


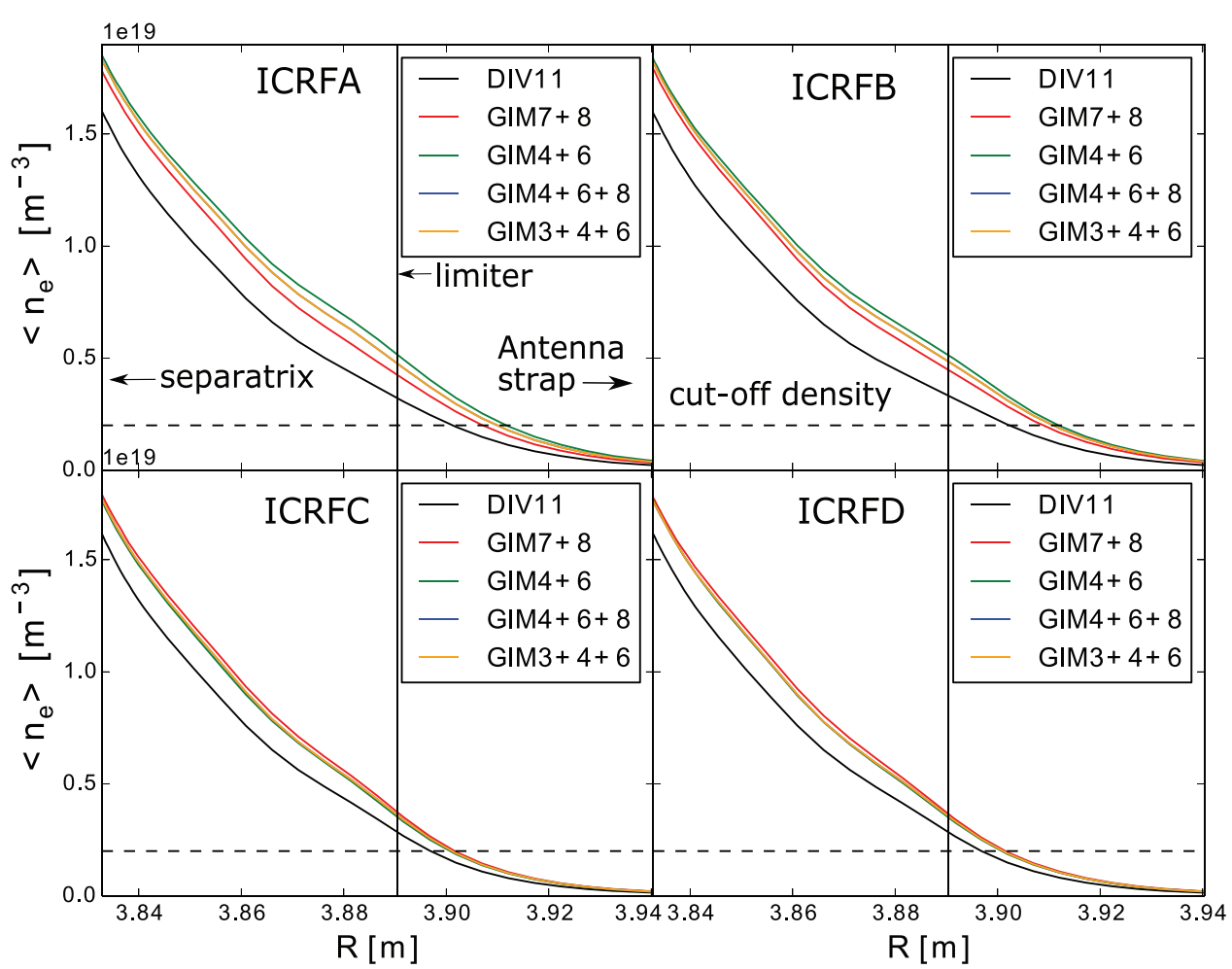

Figure 17. Averaged density in front of the antennas for combined gas puffing scenarios.

that with only one top GIM (GIM7 or GIM8). This is reasonable because the density increase is independent of the top GIMs. The combined two mid-plane GIMs (GIM4 + GIM6) can largely increase the density both in front of antennas $\mathrm{A}$ and $\mathrm{B}$, while the density in front of antennas $\mathrm{C}$ and $\mathrm{D}$ is increased by a much smaller degree. This is because GIM4 is closest to antenna A and GIM6 is closest to antenna B while these two GIMs are both far away from antennas C and D. Compared to the single mid-plane gas puff with GIM4, the density increase in front of antenna A is less significant. This is because only half of the gas is used for GIM4 (the other half is for GIM6). As for the combined mid-plane and top gas puff (GIM4 + GIM6 + GIM8), the density increase in front of antennas A and B is less than case II but larger than case I while the density increase in front of antennas C and D is almost the same for all the three cases.

These simulations show that the combined gas puff can be effective in increasing the SOL density globally. Thus, if good coupling for antennas A and B and ILA are required at the same time, it is best to use GIM4 + GIM6; if good coupling both for antennas $\mathrm{C}$ and $\mathrm{D}$ is desired, one should use GIM3; if good coupling for all the antennas is needed, the best solution would be GIM3 + GIM4 + GIM6.

\section{Conclusions and outlook}

In continuation of a previous study for AUG [8], realistic and comprehensive gas puff simulations were carried out in JET-ILW with the 3D edge plasma fluid and neutral particle code EMC3-EIRENE. In the simulations, we have implemented grid with $360^{\circ}$ toroidal extension and included all the essential plasma facing components, the Gas Injection Modules (GIMs) and the gas pump. Various gas puffing scenarios including the local gas puff and the combined gas puff are investigated. Our simulated electron density and neutral pressure are in quantitative agreement with the experimental ones. The simulations confirm that during mid-plane gas puffing, the edge density is increased most significantly but locally. The largest ICRF coupling increase $(\sim 130 \%)$ is found for the antenna nearest to the mid-plane GIM, and this increase gradually decays with the toroidal distance of the antenna to the gas injection point. 1D RF coupling calculations that use the local density profiles computed by EMC3EIRENE in front of the antennas indicate that the toroidal decay of the RCCR is lower than what was observed in the JET-ILW experiments. The perpendicular particle transport coefficient is shown to have an influence on the magnitude of the RCCR values but not on its toroidal decay length. It is suspected that factors such as the lack of drifts in the EMC3EIRENE code and the simplified physics in the 1D RF coupling code are responsible for this difference. During top gas puffing, the edge density increase is almost toroidally uniform but at smaller magnitude. An almost equal and moderate ICRF coupling increase $(\sim 45 \%)$ is found for all the antennas with top gas puffing.

Our results also indicate that the enhanced SOL density and ICRF coupling are due to the spreading of the injected gas, the local ionization and the magnetic connections from the gas cloud to the antennas. Local ionization effects due to large RF-fields close to the antenna structures were not included in the simulations. To ensure the best ICRF coupling efficiency, using GIMs which can generate local gas magnetically connected to the antennas is important. The simulations also 
confirm that combined gas puff can be effective in increasing the SOL density and the ICRF coupling globally.

The validation of 3D simulations with the EMC3-EIRENE code and the experimental results in AUG [8] and JET-ILW make us confident that similar simulations can be carried out for ITER and other tokamaks. From our studies, it can be inferred that using middle plane gas valves closer to the powered ICRF antenna is the optimized way in terms of maximizing ICRF power coupling. We plan to do similar simulations for ITER and DEMO in the near future.

\section{Acknowledgments}

This work has been carried out within the framework of the EUROfusion Consortium and has received funding from the Euratom research and training programme 2014-2018 under grant agreement No 633053. The views and opinions expressed herein do not necessarily reflect those of the European Commission.

\section{References}

[1] Graham M. et al 2012 Plasma Phys. Control. Fusion 54074011

[2] Lamalle P. et al 2015 Radiofreq. Power Plasmas 1689030007

[3] Stix T.H. 1992 Waves Plasmas (American Institute of Physics)

[4] Bilato R. et al 2005 Nucl. Fusion 45 L5-7

[5] Jacquet P. et al 2016 Nucl. Fusion 56046001

[6] Zhang W. et al 2015 AIP Conf. Proc. 1689050006

[7] Bobkov V. et al 2015 AIP Conf. Proc. 1689030004

[8] Zhang W. et al 2016 Nucl. Fusion 56036007

[9] Jacquet P. et al 2012 Nucl. Fusion 52042002
[10] Lerche E. et al 2015 J. Nucl. Mater. 463 634-9

[11] Saibene G. et al 1999 Nucl. Fusion 39 1133-56

[12] Joffrin E. et al 2014 Nucl. Fusion 54013011

[13] Pinsker R.I. et al 2011 AIP Conf. Proc. 1406313

[14] Pinsker R.I. 2011 37th EPS Conf. on Plasma Physics (Dublin, Ireland, 21-25 June, 2010) vol O4.124 (http://ocs. ciemat.es/EPS2010PAP/pdf/O4.124.pdf)

[15] Ekedahl A. et al 2015 Radiofreq. Power Plasmas 1689030013

[16] Maruyama S. et al 2010 Proc. 23rd Int. Conf. on Fusion Energy (Daejeon) (Vienna: IAEA) CD-ROM file (http:// www-naweb.iaea.org/napc/physics/FEC/FEC2010/html/ fec10.htm)

[17] Maruyama S. et al 2012 Proc. 24th Int. Conf. on Fusion Energy (San Diego) ITR/P5-24 (http://www-naweb.iaea. org/napc/physics/FEC/FEC2012/papers/332_ITRP524.pdf)

[18] Feng Y. et al 2004 Contrib. Plasma Phys. 44 57-69

[19] Colas L. et al 2015 J. Nucl. Mater. 463 735-8

[20] Zhang W. et al 2016 Plasma Phys. Control. Fusion 58095005

[21] Van Eester D. et al 2013 Plasma Phys. Control. Fusion 55025002

[22] Lyssoivan A. et al 2012 Plasma Phys. Control. Fusion 54074014

[23] Tripský M. et al 2014 41st EPS Conf. on Plasma Physics (Berlin, Germany) P1.133 (http://ocs.ciemat.es/ EPS2014PAP/pdf/P1.133.pdf)

[24] Bobkov V. et al 2014 AIP Conf. Proc. 1580271

[25] Jacquet P. et al 2011 Nucl. Fusion 51103018

[26] Jacquet P. et al 2013 J. Nucl. Mater. 438 S379-83

[27] Reiter D. et al 2005 Fusion Sci. Technol. 47 172-86

[28] Feng Y. et al 2006 Nucl. Fusion 46 807-19

[29] Sharma D. et al 2005 Nucl. Fusion 45 825-36

[30] Lunt T. et al 2012 Nucl. Fusion 52054013

[31] Lunt T. et al 2015 J. Nucl. Mater. 463 744-7

[32] Lao L.L. et al 1985 Nucl. Fusion 25 1421-36

[33] Stangeby P.C. 2000 The Plasma Boundary of Magnetic Fusion Devices (Bristol: Institute of Physics Publishing)

[34] Lerche E. et al 2016 Nucl. Fusion 56036022

[35] Jacquet P. et al 2014 Phys. Plasmas 21061510 